\title{
Association among Systolic Blood Pressure Variation, Inflammation and Arterial Rigidity in Essential Hypertension
}

\author{
Caoqian Jiangyan*, Lvwei Zhengliqiang and Mashumei Lixiaodong
}

Sheng Jing Hospital of China Medical University, Shengyang, Liaoning 110004, P.R. China

\begin{abstract}
Objective: To assess the relationship among systolic blood pressure variation (BPV), inflammation and arterial rigidity in essential hypertension.

Methods: This study enrolled 80 patients with essential hypertension, who were started on blood pressure control treatment with medications and lifestyle modification, and 18 normotensive healthy controls. At enrollment and 1-year follow-up, systolic BPV was evaluated by coefficient of variation (CV) in ambulatory blood pressure monitoring; arterial rigidity by brachial-ankle pulse wave velocity (ba-PWV) using ColinVP-1000; and inflammation by high-sensitivity plasma C-reactive protein (hs-CRP) level using rate nephelometry; electrocardiography, blood glucose, lipid profile, creatinine, blood urea nitrogen, and liver and renal function also were assessed.

Results: There were no significant differences in demographics, clinical or biochemical profiles between patients and normotensive controls, except for blood pressure at enrollment but not 1-year follow-up, reflecting effectiveness of blood pressure control measures, with significant decreases in CV, ba-PWV and hs-CRP between enrollment and 1-year follow-up in hypertensive patients; change in CV significantly correlated with those in ba-PWV and hs-CRP (both $P<0.001$, adjusted by SBP d).
\end{abstract}

Conclusion: The effect of systolic BPV on arterial rigidity could be mediated by its influence on the onset and progression of inflammation.

Keywords: Systolic blood pressure variation; High-sensitivity C-reactive protein; Pulse wave variation; Hypertension

\section{Abbreviations}

ABPM: Ambulatory Blood Pressure Monitoring; Ba-PWV: BrachialAnkle Pulse Wave Velocity; BP: Blood Pressure; BPV: Blood Pressure Variation; CV: Coefficient of Variation; DBP: Diastolic Blood Pressure; HDL: High-Density Lipoprotein; Hs-CRP: High-sensitivity C-Reactive Protein; LDL-C: Low-Density Lipoprotein Cholesterol; SBP: Systolic Blood Pressure; TC: Total Cholesterol; TG: Triglyceride

\section{Introduction}

Although the association among arterial rigidity, inflammation and atherosclerosis has been established [1], that among systolic blood pressure variability (BPV, defined as blood pressure variation per unit time [1-3], inflammation and arterial rigidity is yet to be elucidated. The present study, which is the first to report simultaneous measurements of BPV (assessed by coefficient of variation (CV) in ambulatory blood pressure monitoring $(\mathrm{ABPM})$ ), inflammation (as reflected by levels of high-sensitivity C-reactive protein (hs-CRP) and arterial rigidity (assessed by brachial-ankle pulse wave velocity (ba-PWV) at baseline and 1-year follow-up, is aimed at testing the hypothesis that greater blood pressure variation (which is higher among hypertensive than normotensive individuals [4-7] translates into stronger shearing force on the arterial wall, which could trigger inflammation (known to be involved in the pathogenesis and progression of hypertension $[8,9]$ leading to arterial rigidity. The target of antihypertensive therapy therefore would not only be good blood pressure control and improved endothelial function but also BPV lowering $[10,11]$ with consequent decreased inflammation and greater arterial elasticity. The present study was conducted in Northeast China, where essential hypertension, which is associated with increased mortality, is common and daily salt intake is relatively high, approximately $16-18 \mathrm{~g}$, increasing susceptibility to atherosclerosis and more severe cardiovascular diseases [12].

\section{Materials and Methods}

\section{Subjects}

The study enrolled 80 patients with essential hypertension and 18 normotensive healthy controls at the Internal Medicine outpatient department of Sheng Jing Hospital. All hypertensive patients met WHO diagnostic criteria of Essential Hypertension, i.e., measurements thrice of systolic blood pressure (SBP) $\geq 140 \mathrm{mmHg}$ and/or diastolic blood pressure (DBP) $\geq 90 \mathrm{mmHg}$ in at least two different days, and ruling out secondary hypertension, diabetes, chronic renal insufficiency, pulmonary fibrosis or other cardiovascular diseases.

\section{Interventions for blood pressure control}

After enrollment, antihypertensive treatments along with lifestyle modification were prescribed to the 80 eligible patients with essential hypertension, and adjusted fortnightly if SBP was over $140 \mathrm{mmHg}$. Of the 80 patients, $15 \%$ received monotherapy for $\mathrm{BP}$ control, and the remaining received combination therapy. An average of 2.1 drugs was administered; calcium channel blockers (CCBs), angiotensin-converting

*Corresponding author: Caoqian, Sheng Jing, Hospital of China Medical University, Shengyang, Liaoning 110004, P.R. China, Tel: 024-96615-22116; Fax: +86 24 23899651; E-mail: caoq@sj-hospital.org

Received September 07 2015; Accepted September 302015 ; Published October 07, 2015

Citation: Jiangyan C, Zhengliqiang L, Lixiaodong M (2015) Association among Systolic Blood Pressure Variation, Inflammation and Arterial Rigidity in Essentia Hypertension. J Hypertens (Los Angel) 4: 207. doi:10.4172/2167-1095.1000207

Copyright: ( $) 2015$ Jiangyan C, et al. This is an open-access article distributed under the terms of the Creative Commons Attribution License, which permits unrestricted use, distribution, and reproduction in any medium, provided the original author and source are credited. 
Citation: Jiangyan C, Zhengliqiang L, Lixiaodong M (2015) Association among Systolic Blood Pressure Variation, Inflammation and Arterial Rigidity in Essential Hypertension. J Hypertens (Los Angel) 4: 207. doi:10.4172/2167-1095.1000207

Page 2 of 4

enzyme inhibitors (ACE-Is), and angiotensin-receptor blockers (ARBs) accounted for $60 \%, 40 \%$, and $40 \%$ of the antihypertensive agents, respectively.

All patients were advised to modify their lifestyle according to guidelines, including smoking cessation; weight loss; daily alcohol consumption limited to no more than 1 ounce (two drinks) for men and half an ounce (one drink) for women; at least 180 minutes weekly of moderate-intensity physical activity; and no more than $100 \mathrm{mmol}$ daily of dietary sodium.

\section{Study parameters and definitions}

Systolic BPV, ba-PWV, hs-CRP level and dynamic electrocardiography (ECG) were recorded at the time of patient enrollment and at one year follow-up as detailed below. Systolic BPV was determined using a coefficient of variation $(\mathrm{CV})$, which was calculated by dividing the standard deviation of the 24-hour ambulatory SBP by the mean ambulatory SBP. Blood pressure monitoring was carried out using 24-hour ABPM with a noninvasive, portable, BP and ECG monitor by cuff method (Spacelabs Healthcare, Hertford, UK). Oscillometric or auscultatory methods were employed to measure the brachial artery pressure of the left upper limb. The monitoring period spanned from 8 to 9 am until 8 to 9 am of the following day, with measurements taken at 30-minute intervals. The range criteria for effective readings were: SBP, 70 to $260 \mathrm{mmHg}$; DBP, 40 to $150 \mathrm{mmHg}$; pulse pressure, 20 to $50 \mathrm{mmHg}$; and heart rate, 20 to 200 beats per minute. Measured values outside of the latter ranges were considered invalid, and the monitoring device automatically supplied a corresponding reading by re-measurement. If less than $80 \%$ of BP readings in a 24 -hour period were valid, re-monitoring was required starting on the following day. During BP monitoring, patients were asked to maintain their daily routine but to avoid strenuous activities.

Brachial-ankle PWV was measured from humerus to ankle with a Colin VP-1000 screening device (Kyoto, Japan) after patients had rested for 15 to 30 minutes. The right upper arm and the right ankle were measured simultaneously, and distance was used to correct the time difference of pulse wave initiation.

hs-CRP level was determined using rate nephelometry in $2 \mathrm{~mL}$ fasting venous blood drawn in the morning. Lipid panel, blood glucose, and liver and renal function tests were measured as well.

\section{Statistical Analysis}

Continuous variables are presented as mean \pm standard deviation and compared using the paired t-test, while categorical variables are expressed as percentages and were compared using the McNemar's test. Partial correlations, which were adjusted by change in SBP, were used to assess the associations between change in CV change and change in CRP or ba-PWV from baseline to 1-year follow-up. Multiple linear regressions were also used to analyze these associations. Three models were constructed to analyze whether the association between changes in CV and ba-PWV were mediated by change in hs-CRP: in model 1, change in ba-PWV was the dependent variable, and changes in SBP and CV were the independent variables; in model 2, change in hs-CRP was the dependent variable, and changes in SBP and CV were the independent variables; in model 3 , change in ba-PWV was the dependent variable, and changes in SBP, CV and hs-CRP were the independent variables. All analyses were performed with SPSS statistical software version 13.0 (SPSS Inc., Chicago, IL, USA). A P value less than 0.05 was considered statistically significant.

\section{Results}

There were no significant differences in baseline demographics and clinical characteristics between hypertensive patients and normotensive healthy controls (Table 1) or in lipid profile, blood glucose, or liver and renal function tests.

As shown in Table 2, at one-year follow-up, BPV, SBP, ba-PWV and hs-CRP were significantly decreased relative to baseline, and no significant differences were apparent between hypertensive and normotensive groups.

From baseline to 1-year follow-up, change in CV correlated positively with changes in hs-CRP (Figure $1 ; r=0.533, P<0.001$; adjusted by SBP difference) and ba-PWV (Figure 2; $r=0.674, \mathrm{P}<0.001$; adjusted by SBP difference). Positive correlations among changes in CV, hs-CRP and PWV were confirmed in models $1(B=36.260, S . E=4.533, P<0.001)$ and $2(B=0.429, S . E=0.078, P<0.001)$. When changes in CV and hs-CRP were simultaneously integrated into linear regression model 3 , change in hs-CRP lost $(B=3.303, S . E=6.693, P=0.623)$, while change in $C V$ maintained significance with change in ba-PWV $(B=34.844, S . E=5.384$, $P<0.001)$, suggesting that the effect of $\mathrm{CV}$ on ba-PWV was at best partially mediated by its effect on hs-CRP.

\begin{tabular}{|c|c|c|}
\hline \multirow{2}{*}{ Characteristic } & Normotensives & Essential hypertension \\
\cline { 2 - 3 } & $\mathbf{N = 1 8}$ & $\mathbf{N}=\mathbf{8 0}$ \\
\hline Age, years & $60.5 \pm 8.8$ & $61.5 \pm 11.2$ \\
\hline Gender, male/female & $10-$ Aug & $34 / 46$ \\
\hline Smoker, $\mathrm{n}(\%)$ & $5(27.8)$ & $24(30)$ \\
\hline Total Cholesterol, mmol/L & $5.07 \pm 1.65$ & $5.16 \pm 0.58$ \\
\hline Triglyceride, mmol/L & $1.90 \pm 1.30$ & $1.78 \pm 0.95$ \\
\hline LDL-cholesterol, mmol/L & $3.14 \pm 1.19$ & $3.21 \pm 0.74$ \\
\hline HDL-cholesterol, mmol/L & $1.30 \pm 0.36$ & $1.26 \pm 0.35$ \\
\hline $\begin{array}{c}\text { Fasting blood glucose, mmol/L } \\
\text { Systolic blood pressure, } \\
\mathrm{mmHg}\end{array}$ & $5.68 \pm 2.67$ & $5.71 \pm 2.30$ \\
\hline $\begin{array}{c}\text { Diastolic blood pressure, } \\
\mathrm{mmHg}\end{array}$ & $124.7 \pm 15.1$ & $154.6 \pm 8.2$ \\
\hline
\end{tabular}

Table 1: Comparison of baseline characteristics between patients with essential hypertension and normotensive healthy controls.

\begin{tabular}{|c|c|c|c|c|}
\hline Variable & Enrollment & Follow-up & Difference value & $\begin{array}{c}P \\
\text { value* }\end{array}$ \\
\hline hs-CRP, mg/L & $5.56 \pm 1.14$ & $2.92 \pm 1.19$ & $-2.63 \pm 1.22$ & $<0.05$ \\
\hline SBP, mmHg & $154.6 \pm 8.2$ & $128.5 \pm 7.5$ & $-26.1 \pm 7.1$ & $<0.05$ \\
\hline CV & $12.4 \pm 0.9$ & $11.3 \pm 1.3$ & $-1.2 \pm 1.6$ & $<0.05$ \\
\hline $\begin{array}{c}\text { ba-PWV, } \\
\text { cm/s }\end{array}$ & $1605.2 \pm 76.0$ & $1479.1 \pm 80.4$ & $-126.2 \pm 81.5$ & $<0.05$ \\
\hline
\end{tabular}

*paired t test.

Table 2: Changes in study parameters between patient enrollment and 1-year follow-up. 


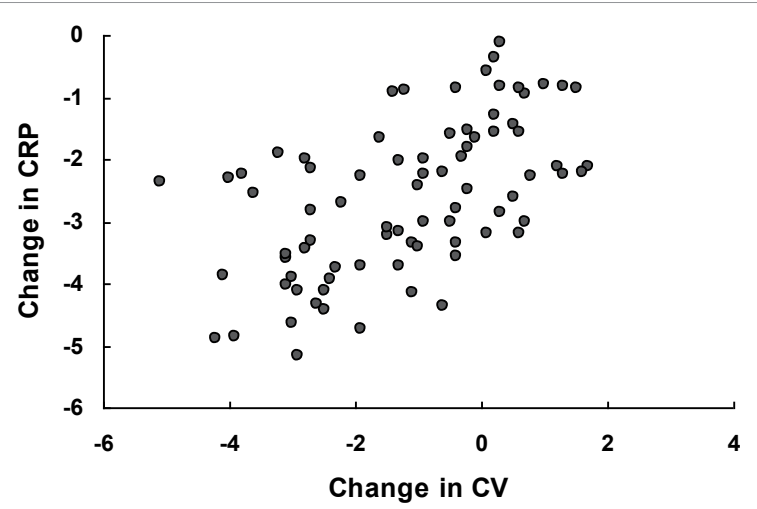

Figure 1: Correlation between changes in CV and hs-CRP ( $r=0.533$ $P<0.001$; adjusted for change in SBP).

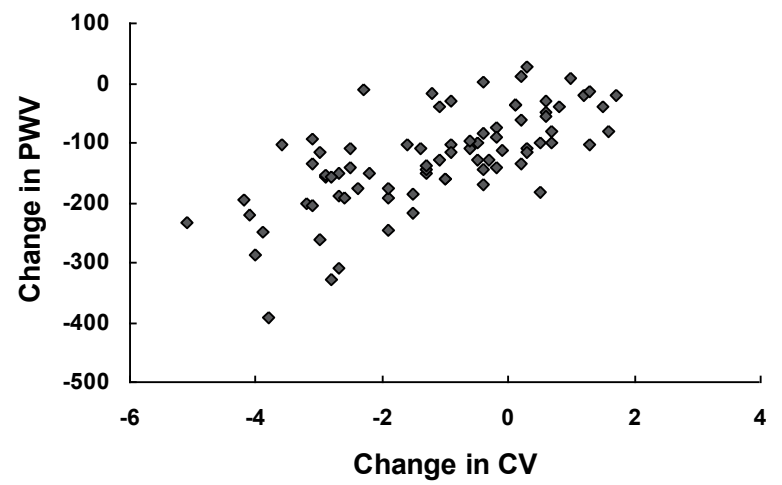

Figure 2: Correlation between changes in $\mathrm{CV}$ and $\mathrm{PWV}(r=0.674, P<0.001$; adjusted for change in SBP).

\section{Discussion}

The present study of Chinese patients with essential hypertension is to our knowledge the first one to simultaneously assess the association among changes in systolic BPV, vascular inflammation and arterial rigidity during 1-year follow-up. The study showed that change in BPV correlated with those in arterial rigidity and vascular inflammation. Good control of BP and improvement in endothelial cell function likely helped to reduce PWV [10], which is consistent with the results from an analysis of daytime and nighttime BP [13].

With a decrease in both $\mathrm{BP}$ and $\mathrm{CV}$ under anti-hypertensive treatment, inflammatory reactions were under control to a certain degree, as evidenced by reduced hs-CRP level. The correlation found in the present study between the change in CV with that in hs-CRP indicates that BPV would be related to inflammation in Chinese hypertensive patients; which might be the case for both normalweight and obese adults $[8,14]$. However, when the associations among changes in CV, hs-CRP, and ba-PWV were studied simultaneously, that between hs-CRP and BPV lost statistical significance while that between ba-PWV and BPV maintained it, which does not rule out a role for inflammation as mediator of vascular rigidity but warrants further study with larger populations and other inflammatory markers.

After the effects of BP were adjusted, BPV increased, which increased vascular inflammation. This finding would have important clinical significance for treatment of hypertensive patients and reduction in target organ damage. The pathophysiology of hypertension includes inflammatory reactions, with elevated hs-CRP levels as a risk factor for primary hypertension [12], and hypertension and inflammation together contribute to an increase in arterial rigidity, which may also facilitate the development of atherosclerosis [15-17] and progression of hypertensive disease generating a vicious cycle [18]. Understanding the relationship between blood pressure and inflammatory reactions is of great significance for both the prevention and treatment of essential hypertension, as well as for the early prevention and treatment of the resulting atherosclerosis.

The present studies have several limitations. First, blood pressure and blood pressure variability has an impact on arterial rigidity. Therefore, we eliminate the impact caused by blood pressure through healthy lifestyle and drugs. However, many kinds of hypertensive drugs may cause different changes in blood pressure variability. In the next study, we are trying to enlarge the number of patients in essential hypertension group to evaluate the impact of different drugs on the blood pressure variability. Second, the effect of the multiple drugs prescribed on systolic BPV was not tested; nevertheless, the study suggests additional benefit of blood pressure management. Third, several methods have been used to evaluate BPV in clinical trials, such as the difference between daytime and nighttime BP, the standard deviation of $\mathrm{BP}$ readings, and the $\mathrm{CV}$; however, the latter appears to more accurately reflect fluctuations of BP and was used in this study. Lastly, although ba-PWV is the gold standard to assess arterial stiffness $[17,19]$, it is affected by many factors such as blood pressure, blood sugar and obesity.

Based on the findings of the present study, systolic BPV might influence the onset and progression of inflammation, thereby further affecting arterial rigidity and facilitating atherosclerosis development, a possibility that remains to be tested. Controlling blood pressure and reducing systolic BPV might help to limit vessel damage, especially arterial rigidity; further study of available medications and development of new ones might individually or in combination [20,21] achieve this goal.

\section{References}

1. Andrikou I, Tsioufis C, Dimitriadis K, Syrseloudis D, Valenti P, et al. (2011) Similar levels of low-grade inflammation and arterial stiffness in masked and white-coat hypertension: comparisons with sustained hypertension and normotension. Blood Press Monit 16: 218-223.

2. Bautista LE, López-Jaramillo P, Vera LM, Casas JP, Otero AP, et al. (2001) Is C-reactive protein an independent risk factor for essential hypertension? J Hypertens 19: 857-861

3. Aalbers $\mathrm{J}$ (2010) Reduced blood pressure variability in ASCOT-BPLA tria favours use of amlodipine/perindopril combination to reduce stroke risk. Cardiovasc J Afr 21: 115

4. Celik A, OzÃßetin M, Yerli Y, Damar IH, KadÄ \pm H, et al. (2011) Increased aortic pulse wave velocity in obese children. Turk Kardiyol Dern Ars 39: 557-562.

5. Wang G (2006) Effects of impaired glucose metabolism on heart rate variability and blood pressure variability in essential hypertension patients. J Huazhong Univ Sci Technolog Med Sci 26: 654-656.

6. Mancia G (1990) Blood pressure variability: mechanisms and clinica significance. J Cardiovasc Pharmacol 16 Suppl 6: S1-6.

7. Kudo H, Kai H, Kajimoto H, Koga M, Takayama N, et al. (2009) Exaggerated blood pressure variability superimposed on hypertension aggravates cardiac remodeling in rats via angiotensin II system-mediated chronic inflammation. Hypertension 54: 832-838.

8. Abramson JL, Lewis C, Murrah NV, Anderson GT, Vaccarino V (2006) Relation of $\mathrm{C}$-reactive protein and tumor necrosis factor-alpha to ambulatory blood pressure variability in healthy adults. Am J Cardiol 98: 649-652. 
Citation: Jiangyan C, Zhengliqiang L, Lixiaodong M (2015) Association among Systolic Blood Pressure Variation, Inflammation and Arterial Rigidity in Essential Hypertension. J Hypertens (Los Angel) 4: 207. doi:10.4172/2167-1095.1000207

9. Harada S, Takeda K (2004) Pulse wave velocity (PWV) [Article in Japanese]

10. Harada S, Takeda K (2004) [Pulse wave velocity(PWV)]. Nihon Rinsho 62: 1136-1142.

11. Rafidah HM, Azizi A, Noriah MN (2008) Blood pressure variability and arterial elasticity in hyperlipidaemic subjects. Singapore Med J 49: 297-303.

12. Figueiredo VN, Yugar-Toledo JC, Martins LC, Martins LB, de Faria AP, et al. (2012) Vascular stiffness and endothelial dysfunction: Correlations at different levels of blood pressure. Blood Press 21: 31-38

13. Dong G, Sun Z, Zheng L, Li J, Zhang X, et al. (2007) Prevalence, awareness, treatment, and control of hypertension in rural adults from Liaoning Province, northeast China. Hypertens Res 30: 951-958.

14. Nichols WW, O'Rourke MF (2005) McDonald's Blood Flow in Arteries. London: Hodder Arnold.

15. Gupta AK, Cornelissen G, Greenway FL, Dhoopati V, Halberg F, et al. (2010) Abnormalities in circadian blood pressure variability and endothelial function: pragmatic markers for adverse cardiometabolic profiles in asymptomatic obese adults. Cardiovasc Diabetol 9: 58.
16. Ozawa M, Tamura K, Okano Y, Matsushita K, Ikeya Y, et al. (2009) Blood pressure variability as well as blood pressure level is important for left ventricular hypertrophy and brachial-ankle pulse wave velocity in hypertensives. Clin Exp Hypertens 31:669-679.

17. Weber T, Auer J, Lamm G, O'Rourke MF, Eber B (2007) Arterial stiffness, central blood pressures, and wave reflections in cardiomyopathy-implications for risk stratification. J Card Fail 13: 353-359.

18. O'Rourke MF, Staessen JA, Vlachopoulos C, Duprez D, Plante GE (2002) Clinical applications of arterial stiffness; definitions and reference values. Am J Hypertens 15: 426-444

19. Laurent S, Cockcroft J, Van Bortel L, Boutouyrie P, Giannattasio C, et al. (2006) Expert consensus document on arterial stiffness: methodological issues and clinical applications. Eur Heart J 27: 2588-2605.

20. Ichihara A, Kaneshiro Y, Takemitsu T, Sakoda M, Hayashi M (2006) Ambulatory blood pressure variability and brachial-ankle pulse wave velocity in untreated hypertensive patients. J Hum Hypertens 20: 529-536.

21. Xie HH, Shen FM, Xu LP, Han P, Miao CY, et al. (2007) Reduction of blood pressure variability by combination therapy in spontaneously hypertensive rats. J Hypertens 25: 2334-2344. 